\title{
Particle Focusing in Curved Microfluidic Channels
}

\section{SUBJECT AREAS:}

BIOMEDICAL

ENGINEERING

APPLIED PHYSICS

Received

1 October 2013

Accepted

11 November 2013

Published

26 November 2013

Correspondence and requests for materials should be addressed to

M.T. (mtoner@hms. harvard.edu)

\author{
Joseph M. Martel ${ }^{1,2}$ \& Mehmet Toner ${ }^{2}$
}

'School of Engineering and Applied Sciences, Harvard University, Cambridge, Massachusetts, 02 138, USA, ${ }^{2}$ BioMEMS Resource Center, Center for Engineering in Medicine and Surgical Services, Massachusetts General Hospital and Harvard Medical School, Boston, Massachusetts, 02114 , USA.

The decoupled effects of Reynolds and Dean numbers are examined in inertial focusing flows. In doing so, a complex set of inertial focusing behavioral regimes is discovered within curved microfluidic channels over a range of channel Reynolds numbers, curvature ratios and particle confinement ratios. These regimes are characterized by particle migration either towards or away from the center of curvature as the channel Reynolds number is increased. The transition between these two regimes is shown to be a set of conditions where single-point equilibrium position focusing of particles of different sizes is achieved. A mechanism describing the observed motion of particles in such flows is hypothesized incorporating the redistribution of the main flow velocities caused by Dean flow and its effect on the balance forces on suspended particles.

nertial focusing is an area of significant interest in the realm of microfluidics because it combines high throughput capability with precision particle positioning and offers theoretical intrigue due to the seemingly endless surprises that accompany experimental results. Inertial focusing occurs under certain conditions as particles flowing through a microchannel migrate across streamlines to equilibrium positions within the flow. This migration is due to inertial effects of the fluid motion around the particle and the interaction of this flow field with the walls of the channel ${ }^{1-5}$. Equilibrium positions arise from a balance between two distinct effects; a shear gradient lift force directed towards the walls of the channel and an opposing wall effect ${ }^{6,7}$. These forces allow for the precise alignment of particles in a flow at throughputs orders of magnitudes higher than in previous microfluidic technologies. The high throughput nature of inertial focusing has enabled a range of microfluidic technologies for biomedical applications from separation technologies ${ }^{8-12}$, to automated sample preparations ${ }^{13,14}$, to novel cell analysis techniques such as cell deformability cytometry ${ }^{15}$ and the isolation of circulating tumor cells from blood ${ }^{16,17}$.

It is generally accepted that inertial focusing in straight channels is dependent on two main parameters: Reynolds number, defined as $\operatorname{Re}_{\mathrm{C}}=\rho \mathrm{U}_{\text {Max }} \mathrm{D}_{\mathrm{h}} / \mu$, where $\rho$ is the fluid density, $\mu$ is the fluid viscosity, $\mathrm{U}_{\mathrm{Max}} \cong$ $3 / 2 \mathrm{U}_{\mathrm{Avg}}$ is the maximum velocity of the fluid and $\mathrm{D}_{\mathrm{h}}$ is the hydraulic diameter of the channel defined as $\mathrm{D}_{\mathrm{h}}=$ $2 h w /(h+w)$ where $h$ and $w$ are the height and width of the channel cross section respectively, and the particle confinement ratio, $\lambda=a / \mathrm{D}_{\mathrm{h}}$, where $a$, is the particle size. Prior research has determined a minimum threshold for inertial focusing to occur such that $\lambda>0.07$ and $\operatorname{Re}_{C} \lambda^{2}$, also known as the particle Reynolds number, $\operatorname{Re}_{\mathrm{P}}$, is $\geq 1^{18}$. The equilibrium positions can be further controlled using curved channels, where a secondary flow called Dean flow is established at finite $\operatorname{Re}_{\mathrm{C}}$. The strength of this secondary flow is characterized by the inertia of the fluid and the curvature of the channel, or the non-dimensional Dean number defined as $D e=\operatorname{Re}_{\mathrm{C}} \delta^{1 / 2}$, where $\delta$ is the curvature ratio $\delta=D_{h} / 2$, where $r$ is the radius of curvature of the channel. This secondary flow imparts an additional drag force on particles thereby adjusting the number and locations of inertial focusing equilibrium positions $\mathrm{s}^{919-30}$. A review of these effects and the field of inertial microfluidics in general is available elsewhere ${ }^{18}$.

While the majority of the biomedical applications of inertial focusing utilize curved channels, the complex nature of the flow has been a barrier to detailed mathematical description and therefore much of the development in curved channels has followed an empirical approach. Several of these empirical studies have been completed about curved inertial focusing channels investigating different curve designs ${ }^{21,26}$, scalability ${ }^{28,29}$ and dynamics of the focusing behavior ${ }^{9,25}$. The majority of these studies incorporated some method of varying the balance of inertial lift and Dean drag forces, most often accomplished by changing flow rate $e^{19,23,24,27,28,30,31}$ but also by changing the cross section ${ }^{24,22,25}$, and investigating multiple lanes of a spiral with different curvature values ${ }^{9,25}$. However, every one of these previous studies have varied both important non-dimensional parameters $\left(\operatorname{Re}_{\mathrm{C}}\right.$ and De) simultaneously or measured the particle behavior for different residence times at a given flow rate, which has been shown to affect eventual focusing behavior ${ }^{25}$. In order to develop a more complete understanding of the 
relative significance of the dominant forces for inertial focusing in curved channels, it is necessary to study the effect of Reynolds and Dean numbers independently. In this report, we present a systematic study of the decoupled effects of Dean number and Reynolds number on inertial focusing behavior for a range of different particle confinement ratios. These results are then interpreted in terms of the potential to separate particles exposing different regimes of particle migration in curved channels.

\section{Results}

Lateral focusing at varied Reynolds and Dean numbers. In order to decouple the effects of Reynolds and Dean numbers in inertial focusing flows it was necessary to create a set of devices with different radii of curvature. These devices were then tested at similar flow conditions with three different particle sizes and measuring the particle distribution at the outlets. $\operatorname{Re}_{C}$ maps are used to present the results of the parameter space exploration ${ }^{21}$. These have been compiled by taking intensity line scans across images taken at the outlet position of each channel at different flow rates. Each line scan becomes a column of pixels in the $\operatorname{Re}_{\mathrm{C}}$ map showing the inertial focusing behavior at the outlet across a large range of $\operatorname{Re}_{\mathrm{C}}$ values. The bottom of each $\operatorname{Re}_{\mathrm{C}}$ map represents the inside wall of the channel closer to the center curvature $(\mathrm{x} / w=0)$ and the top is the outside wall, further from the center of curvature $(\mathrm{x} / w=1)$. SI Figure 1 gives a graphical explanation for the creation of these plots.

Figure 1 shows a compilation of the particle focusing behavior over the investigated ranges of the three key variables: $\operatorname{Re}_{C}, \delta$, and $\lambda$. The top row of $\operatorname{Re}_{\mathrm{C}}$ maps shows the behavior of each particle size in a straight channel with an aspect ratio of $h / w=0.5$. As expected, the large particles whose $\operatorname{Re}_{\mathrm{P}}$ values are greater than 1 for $\operatorname{Re}_{\mathrm{C}}>45(\lambda=$ $0.149)$ and $\operatorname{Re}_{\mathrm{C}}>20(\lambda=0.225)$, both focus to the center of the channel width. In comparison, the smaller particles $(\lambda=0.066)$ which do not experience as strong of lift forces since the shear gra- dient lift scales with $a^{3}$, do not achieve as significant of focusing over the same channel length ${ }^{31}$. The smaller particles achieve $\operatorname{Re}_{\mathrm{P}}=1$ at and $\operatorname{Re}_{\mathrm{C}}$ value of 229, and show some focusing trends near the high shear regions near the sides of the channel at lower $\operatorname{Re}_{C}$ values.

These high shear regions seem to retain a small fraction of particles near the sidewalls as $\lambda$ increases at increasing $\operatorname{Re}_{C}$, indicating that the lateral lift forces due to the shear gradient have become strong enough to resist edge effects from the side walls at least over a channel length of $4 \mathrm{~cm}$. For example, these side streaks emerge at certain $\operatorname{Re}_{\mathrm{C}}$ values that scale with $\sim \lambda^{0.4}(\sim 105$ for $\lambda=0.066, \sim 140$ for $\lambda=0.149$ and $\sim 180$ for $\lambda=0.225$ ). The existence of these side streaks also concurs with the decrease in positive lift coefficient found at increasing $\operatorname{Re}_{\mathrm{C}}{ }^{33-35}$. The formation of these side streaks also indicates the limited distance over which the lateral wall effect forces are dominant.

The second row of $\operatorname{Re}_{C}$ maps are for the slightly curved channels and each consecutive row increases in curvature ratio, $\delta$, to the tightest spiral on the bottom row. The Dean number in this tightest spiral device $(\delta=0.0166)$ will increase fastest with respect to $\operatorname{Re}_{C}$ and more slowly as the $\delta$ decreases as shown in SI Figure 2. A direct comparison of the focusing behavior for each size particle at equivalent Reynolds numbers but different Dean numbers is accomplished by comparing a single column of pixels across the $\operatorname{Re}_{\mathrm{C}}$ maps vertically.

For the smallest particles $(\lambda=0.066)$ the addition of a small amount of curvature and the resulting Dean flow causes advection of particles across the channel in a manner similar to Dean flow fractionation $^{24,27}$. This increases the number of particles found at an inner equilibrium position $(\sim 0.1 \mathrm{x} / w)$, but over the $4 \mathrm{~cm}$ channel length does not produce a single lateral equilibrium position. At higher curvature values a single lateral focusing position emerges. At this low confinement ratio $(\lambda=0.066)$ the particles do not initially focus to the center of the channel width. Instead the initial
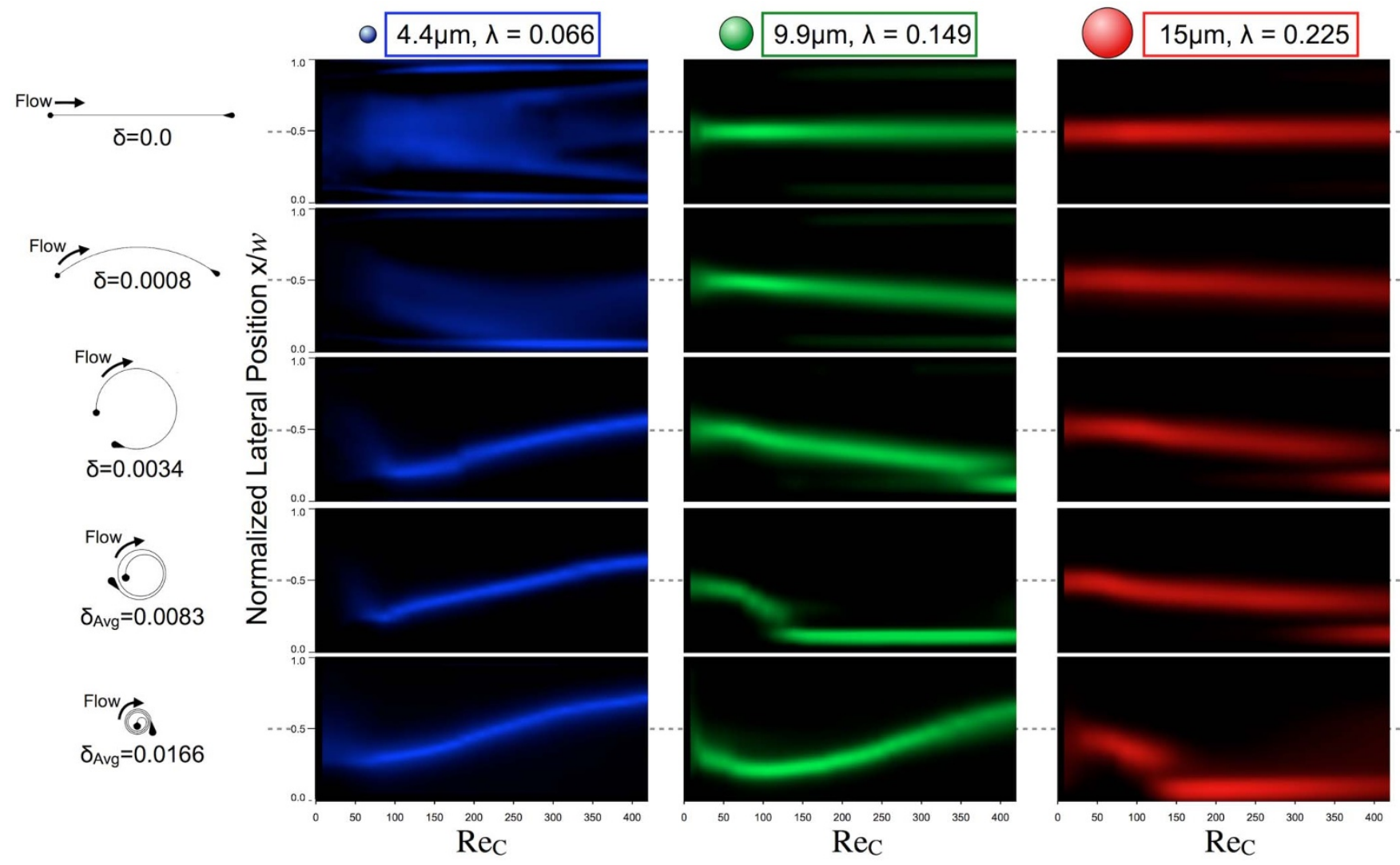

Figure $1 \mid \mathrm{Re}_{\mathrm{C}}$ maps for each condition of curvature (increasing from top row to bottom row) and particle confinement (increasing from left column to right column). Each column of pixels compares equivalent $\mathrm{Re}_{\mathrm{P}}$ at five different Dean numbers. 

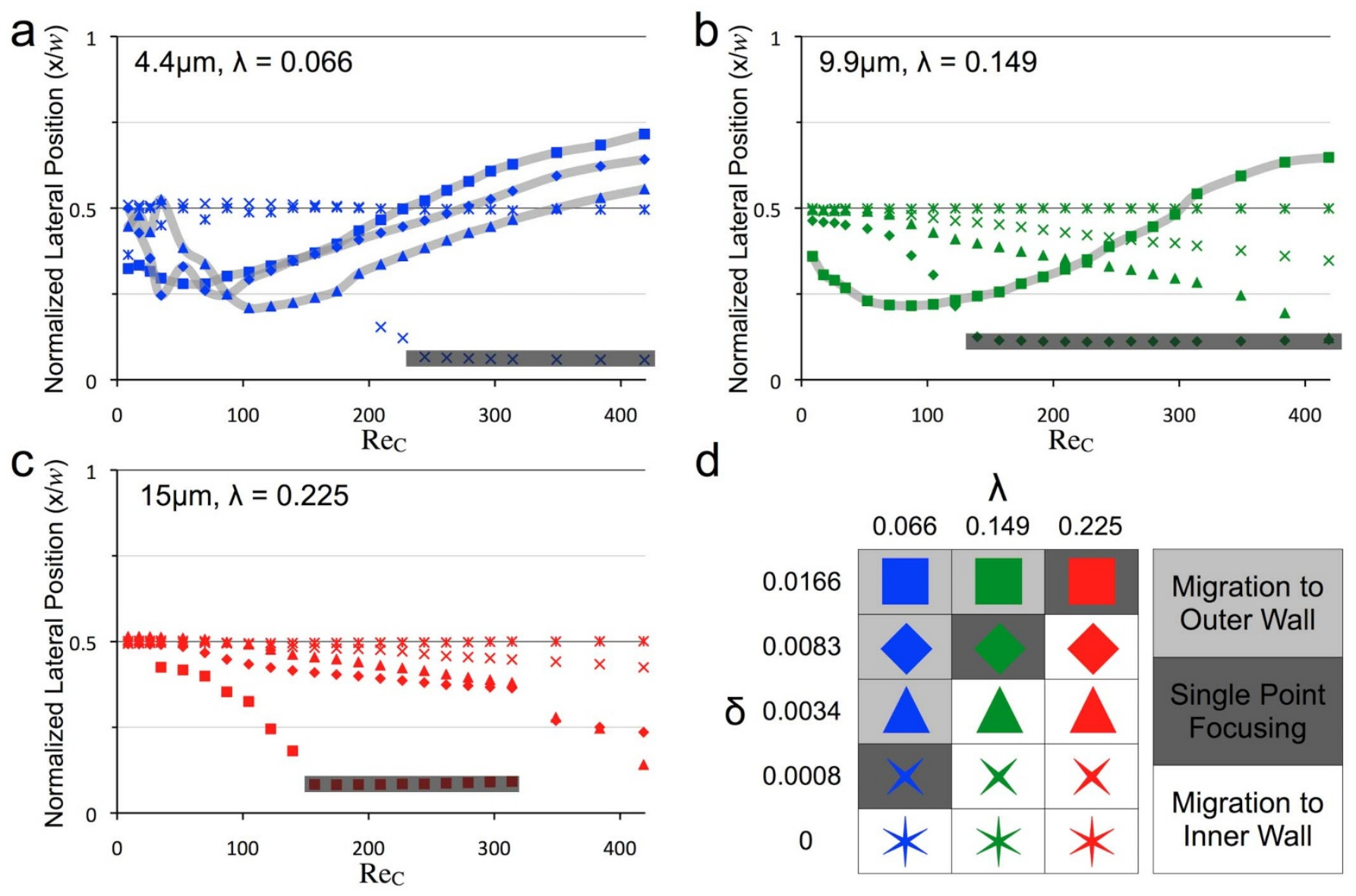

Figure $2 \mid$ Streak position data for all channels plotted versus $\operatorname{Re}_{\mathrm{C}}$ for (a) $\lambda=0.066$ (blue), (b) $\lambda=0.149$ (green) and (c) $\lambda=0.225$ (red). In each plot the marker shape corresponds to the $\delta$ value (asterix $=$ straight, $\mathrm{x}=0.0008$, triangles $=0.0034$, diamonds $=0.0083$ and squares $=0.0166$ ) as shown in the legend (d). Light gray highlights indicate that the specified condition describes migration towards the outside wall. Dark gray highlights indicate single lateral point focusing at innermost equilibrium position.

focusing position is dependent on the curvature. At a $\operatorname{Re}_{\mathrm{C}}$ of 104 the three highest curvature ratio channels $(\delta=0.0034,0.0083,0.0166)$ have achieved single lateral point focusing and the streaks are located at $\mathrm{x} / w=0.210,0.292$ and 0.314 respectively. Qualitatively, there also seems to be a weak trend that the minimum $\operatorname{Re}_{C}$ value to achieve single lateral point focusing decreases with increasing curvature, however, the minimum $\operatorname{Re}_{\mathrm{C}}$ value only varies over the difference between two consecutive flow rates.

For the middle sized particles in the center column of Figure $1(\lambda$ $=0.149$ ), the focused stream initially forms at the centerline of the channel at low $\operatorname{Re}_{\mathrm{C}}(\sim 9)$ in all the channels except the highest curvature where the streak initially forms at $\mathrm{x} / w=0.36$ at the same low $\operatorname{Re}_{\mathrm{C}}(\sim 9)$. As the curvature is increased the central streak shifts towards the inner wall as expected for particles focused to mirrored vertical equilibrium positions at the top and bottom of the channel. A high quality single lateral focusing position is achieved in the $\delta=$ 0.0083 device over $\mathrm{Re}_{\mathrm{C}}$ range of 157-384 and within this range the streak is positioned at approximately $\mathrm{x} / w=0.1$. In the highest curvature case the streak initially forms slightly closer to the inner wall of the curve rather than the centerline, approaches the inner most equilibrium position (minimum $\mathrm{x} / w=0.24$ ) but then the streak moves away from the center of curvature. The largest particles $(\lambda=0.225)$, act similarly to the middle sized particles $(\lambda=0.149)$ although the transition to the innermost equilibrium position is delayed in terms curvature ratio $(\delta=0.0166$ for $\lambda=0.225$ rather than $\delta=0.0083$ for $\lambda=0.149)$.

Through the quantification of streak position ${ }^{25}$, two segregated regimes emerge: 1 , Particles initially focusing at the center of the channel $(\mathrm{x} / w=0.5)$ and migrate towards the inner wall of the curved channel $(\mathrm{x} / w=0)$ with increasing $\operatorname{Re}_{\mathrm{C}}$, and 2 , Particles that initially focus on the inner half of the channel $(\mathrm{x} / w<0.5)$ and eventually migrate towards the outer wall as $\operatorname{Re}_{C}$ increases. Figure 2 presents the two different scenarios and their associated streak position data.

The transition between the two different regimes of focusing appears similar for each particle size. In such flow conditions the streak motion towards the center of curvature jumps to the inner most equilibrium position $(\mathrm{x} / w \sim 0.1)$ as previously mentioned for the $\delta=0.0083, \lambda=0.149$ case. At higher curvature, the streak never reaches this innermost equilibrium position at any $\operatorname{Re}_{C}$ value. One interesting note here is that the transition between regimes occurs at approximately a ratio of $\lambda / \delta^{1 / 2} \sim 2$ for the current set of devices. This can be visualized by measuring the minimum streak position or closest approach to the inner wall for each of the devices at any $\operatorname{Re}_{\mathrm{C}}$ value and plotting versus $\lambda / \delta^{1 / 2}$ as shown in SI Figure 3. For the current device cross section of $100 \mu \mathrm{m}$ wide and $50 \mu \mathrm{m}$ high, a device with $\lambda / \delta^{1 / 2}>2$ will cause focusing behavior towards the inner wall of the curved channel, a device with $\lambda / \delta^{1 / 2}<2$ will cause focusing behavior towards the outer wall of the curved channel. This ratio can therefore be used as a design parameter for achieving the different focusing behaviors described.

Since the three main parameters were varied independently, it is possible to use best-fit analysis to try to collapse the data using the independent parameters. Through this analysis it was determined that the streak position within the two separate regimes (inner and outer directed migration of the streak with increasing $\operatorname{Re}_{\mathrm{C}}$ ) could be nearly linearized although in some cases there were not enough $\lambda$ and $\delta$ combinations to do a complete fit of all three parameters. 

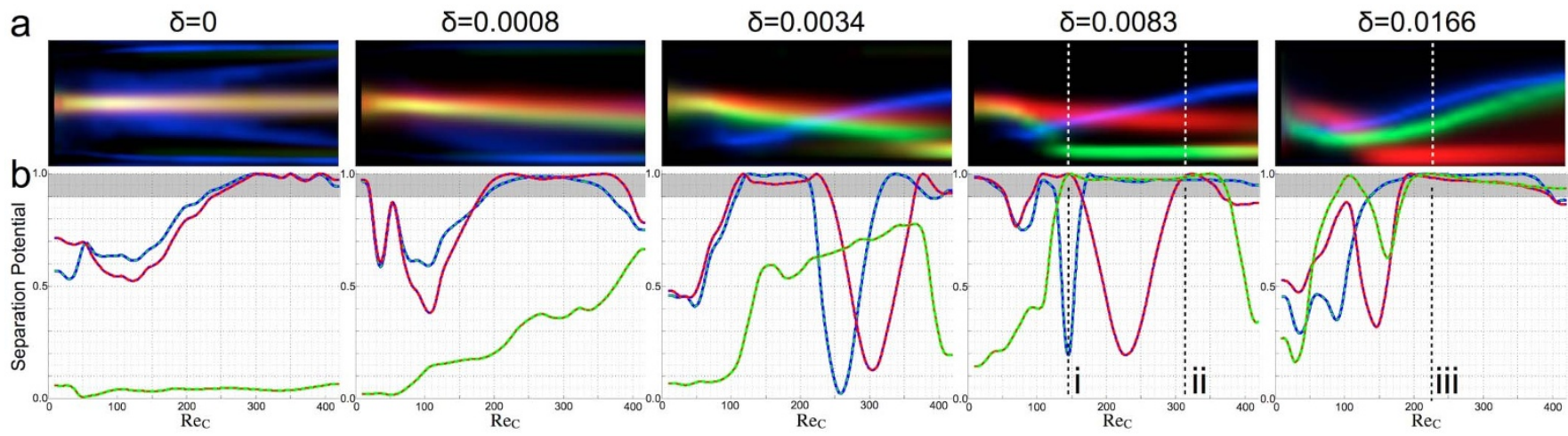

\begin{tabular}{|c|c|}
\hline $\begin{array}{c}5 \text { um } \\
\lambda=0.066\end{array}$ & $\begin{array}{c}\text { 10um } \\
\lambda=0.149\end{array}$ \\
\hline $\begin{array}{c}5 \text { um } \\
\lambda=0.066\end{array}$ & $\begin{array}{c}\begin{array}{c}15 \text { um } \\
\lambda=0.225\end{array} \\
\end{array}$ \\
\hline \multicolumn{2}{|c|}{$-1,-1$} \\
\hline $\begin{array}{c}\text { 10um } \\
\lambda=0.149\end{array}$ & $\begin{array}{c}15 \text { um } \\
\lambda=0.225\end{array}$ \\
\hline
\end{tabular}
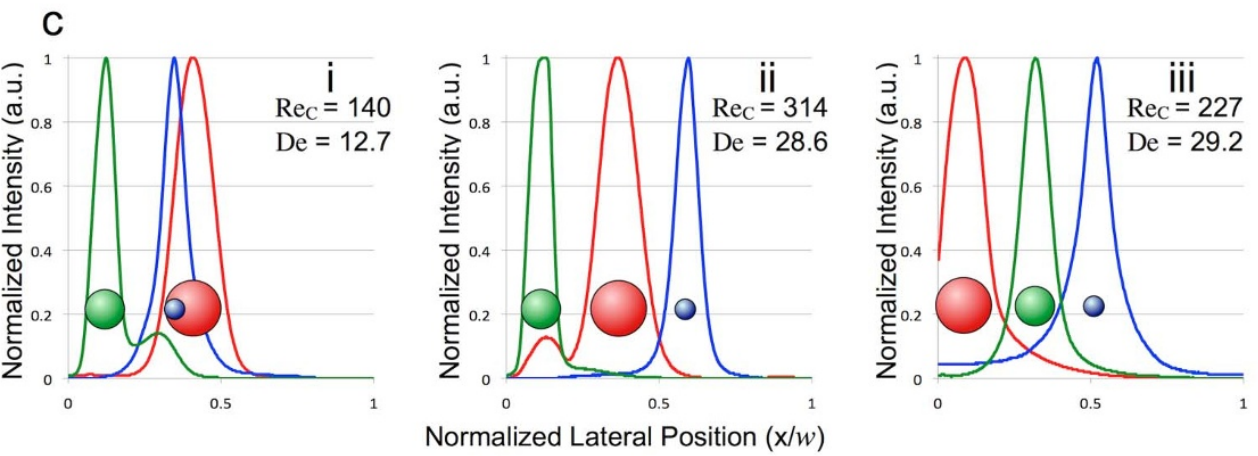

Figure $3 \mid$ (a) This figure merges the previous $\mathrm{Re}_{\mathrm{C}}$ maps for each device for more direct comparison of the effect of particle confinement. (b) These maps are presented above their corresponding separation potential plots. This separation potential is a correlation coefficient calculated as $1-\mathrm{R}^{2}$ between the intensity profiles (distribution of particles) at each flow condition tested. A value of 1 indicates no correlation between particle distributions whereas a value of 0 indicates the distributions are perfectly overlapping. Excellent separation is considered when the separation potential value is greater than 0.9 (grayed area). The three lines on each plot describe the separation potential between each pair of particle sizes (green with red dash represents the separation potential between $\lambda=0.149$ and $\lambda=0.225$, blue with green dash represents the separation potential between $\lambda=0.066$ and $\lambda=0.149$, and red with blue dash represents the separation potential between $\lambda=0.066$ and $\lambda=0.225$ ) as shown in the legend on the bottom left. (c) Intensity line plots are given for selected flow conditions where the arrangement of particle sizes from inside wall to outside wall changes.

Beginning with the $\lambda / \delta^{1 / 2}>2$ regime, the streak position could be collapsed to a single line for each particle size using Dean number alone, $\operatorname{Re}_{\mathrm{C}} \delta^{1 / 2}$. These are cases describing relatively low Dean number flows where the larger particles are shifting towards the inner wall with increasing $\operatorname{Re}_{C}$ but generally do not begin to transition into the single innermost equilibrium position at the inside wall of the channel. Each particle size had a distinct slope $(\lambda=0.149$ slope $=0.012$, $\lambda=0.225$ slope $=0.007$ ), which decreased with increasing particle confinement. Devices designed in this regime can be utilized for particle fractionation $\left(\lambda / \delta^{1 / 2}>2\right.$ for all particle sizes), as the motion is predictable and varies strongly with particle size.

For the $\lambda / \delta^{1 / 2}<2$ regime, the motion of the streak in all cases seem to follow similar paths relative to $\operatorname{Re}_{\mathrm{C}}$ but start at different initial positions dependent on $\lambda$ and $\delta(\lambda=0.149 \delta=0.129$ minimum $\mathrm{x} / \mathrm{w}$ $=0.216, \lambda=0.066 \delta=0.129$ minimum $\mathrm{x} / w=0.260, \lambda=0.066 \delta=$ 0.091 minimum $\mathrm{x} / w=0.248, \lambda=0.066 \delta=0.058$ minimum $\mathrm{x} / \mathrm{w}=$ $0.210)$. In this regime the streak position was less dependent on curvature instead being fit best with $\operatorname{Re}_{C} \delta^{1 / 4}$. Again, these paths had certain slopes for each particle size with the caveat of only a single curvature value for the $\lambda=0.149$ particle size $(\lambda=0.066$ slope $=0.0045, \lambda=0.149$ slope $=0.0052$ ). This slope was measured over the region where the streak is passing the midline of the channel width $(\mathrm{x} / w=0.5)$. This scaling indicates that the higher $\delta$ values cause equilibrium positions closer to the outside wall of the channel curvature at equivalent $\operatorname{Re}_{\mathrm{C}}$ values. This is surprising because if the particles are at equilibrium positions mirrored at the top and bottom of the channel at a consistent height location the Dean flow should be pushing them to the inside wall of the channel curvature at any flow condition. If all particles are below the threshold, $\lambda / \delta^{1 / 2}<2$, this regime is also effective for particle separation.
The transition between these two regimes, when $\lambda / \delta^{1 / 2} \sim 2$, is the only regime overlapped by all three particle confinement ratios, but was only seen at a single curvature ratio for each particle size. The relationships with $\operatorname{Re}_{C}$ and $\delta$ were non-obvious but were approximated as linear with the average Dean velocity in the channel crosssection for the different devices and flow conditions. Each device follows a separate power law in terms of the relationship between the average Dean flow velocities and the average main flow velocity as shown in SI Figure 4 . With this scaling, the data was then best-fit with particle confinement leading to an overall scaling of $\left(\operatorname{Re}_{C} \delta^{1 / 2}\right)^{\mathrm{b}} \lambda^{-1.22}$. When this parameter is approximately equal to 1200 and $\lambda / \delta^{1 / 2} \sim 2$, the streak achieves the closest approach to the inner wall which coincides with the best focusing in terms of the width of the streaks across all conditions tested. This combination of design and operational conditions can be helpful in determining the optimal flow rate for a given device design and particle size. These regimes and associated scalings are summarized in SI Figure 3.

Separation potential and regimes of separation. An intriguing consequence of the aforementioned regimes of focusing is that the arrangement of particles by size across the channel width changes. Not only do these changes indicate a more complex scaling of forces than provided in the literature but there are also implications of these spatial arrangements in terms of the ability to separate particles. The overlaid $\operatorname{Re}_{\mathrm{C}}$ maps in Figure 3a graphically show the streak positions for the different confinement ratios over the entire curvature range. At different flow conditions these intensity distributions are either distinct, able to be separated, or overlapping, unable to be separated. The three highlighted examples (i, ii and iii) present three different cases where the particle streaks are changing in spatial arrangement 
a

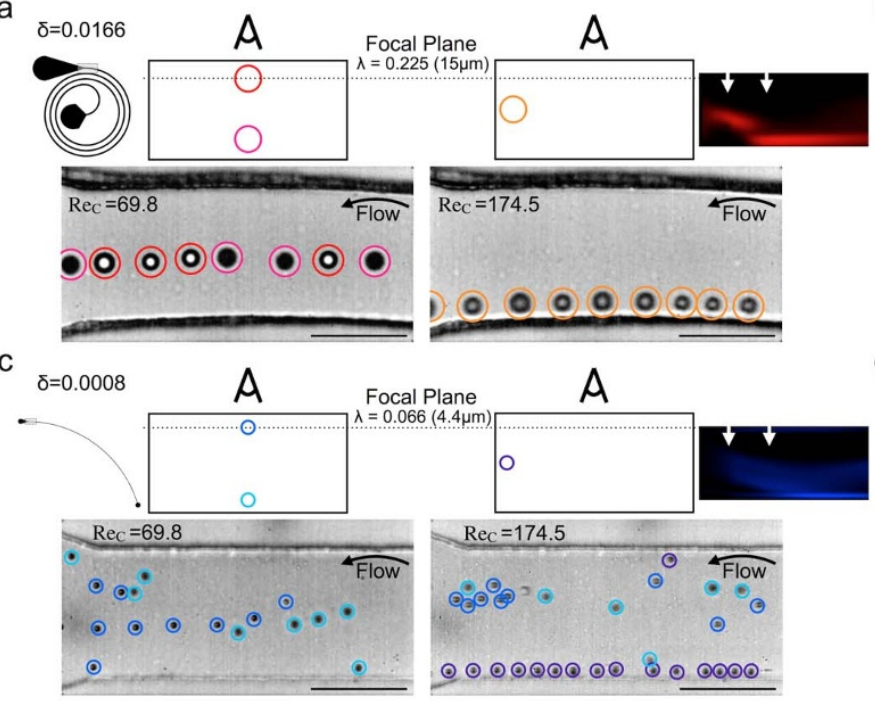

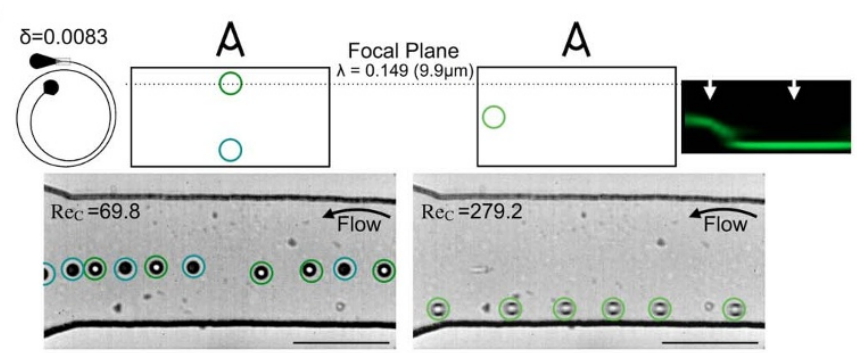

d

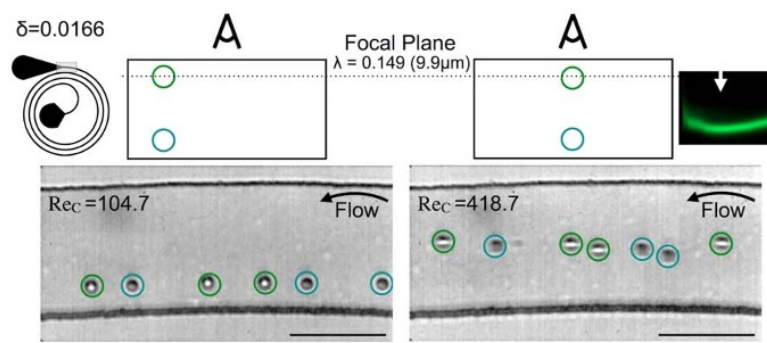

Figure $4 \mid$ This figure shows example high-speed video stills ( $2 \mu$ s exposure time) for different flow conditions below (left) and above (right) the $\operatorname{Re}_{\mathrm{C}}$ value necessary in order to achieve single point focusing at the inside wall for each of the three particle sizes (a) $\lambda=0.225$, (b) $\lambda=0.149$, (c) $\lambda=\mathbf{0 . 0 6 6}$ with associated schematics of the channel cross-sections with assumed vertical positions. $\operatorname{Re}_{\mathrm{C}}$ maps for each device are given with white arrows pointing to the flow conditions being shown with high-speed images. (d) Example high-speed video stills for the $\lambda=0.149, \delta=0.0166$ case where the single point focusing is bypassed and two vertical equilibrium positions always exist. Scale bars are $80 \mu \mathrm{m}$.

across the width of the channel from the inside wall to the outside wall. These cases correspond to the changes in focusing behavior from inner to outer motion of the streak with increasing $\operatorname{Re}_{\mathrm{C}}$ for the different particle sizes. In the $\delta=0.0083$ device alone two different arrangements are achieved by simply changing flow rate. At $\operatorname{Re}_{\mathrm{C}}=140$ (Case i) the order of particles is $\lambda=0.149$ (medium), $\lambda$ $=0.066$ (small) and $\lambda=0.225$ (large) from inside to outside. However, at $\operatorname{Re}_{\mathrm{C}}=314$ (Case ii) the order of particles changes to $\lambda$ $=0.149$ (medium), $\lambda=0.225$ (large) and $\lambda=0.066$ (small) from inside to outside as the larger particles continue to migrate closer to the inside wall and the smaller particles are shifting towards the outside. Finally, in the $\delta=0.0166$ device, both the small and medium particle sizes trend towards the outer half of the channel and the largest particles shift into the innermost equilibrium position allowing for the final order of particles $\lambda=0.225$ (large), $\lambda=0.149$ (medium) and $\lambda=0.066$ (small) at $\operatorname{Re}_{\mathrm{C}}>150$ (Case iii).

A more quantitative assessment of the potential for the separation of the particle sizes presented here is summarized in Figure $3 \mathrm{~b}$ using a correlation coefficient $\left(1-\mathrm{R}^{2}\right)$ between the intensity profiles for each particle size at each of the flow conditions. The graphs from left to right in Figure $3 \mathrm{~b}$ increase in curvature value and have three lines depicting the separation potential between the different combinations of particles. The straight channel $(\delta=0)$ allows for minimal separation between the larger particles since the focus to the same locations at the center of the channel width. The separation between the small particles and both larger particles are therefore closely related and only reach a significant value at higher $\operatorname{Re}_{C}$ when the small particle begin to exhibit five lateral streaks, four of which do not intersect with the distributions of larger particles and thus there is a higher separation potential.

When $\delta=0.0008$, the high separation value is further improved since the small particles begin to exhibit single point lateral focusing near the inside wall. At higher $\mathrm{Re}_{\mathrm{C}}$, the small particles not focused into the innermost equilibrium position begin migrating to the outer half of the channel and overlap with the larger particle streaks leading to a dip in separation potential. Further increasing the curvature to $\delta$ $=0.0034$ is when the small $(\lambda=0.066)$ particles begin to focus initially on the inner half and the streak shifts to the outer half of the channel with increasing $\mathrm{Re}_{\mathrm{C}}$. This occurs simultaneously with the increased migration of the larger particles towards the inside wall.
The separate dips in separation potential between the $\lambda=0.066$ and $\lambda=0.149$ particles and at slightly higher $\operatorname{Re}_{\mathrm{C}}$ between the $\lambda=0.066$ and $\lambda=0.225$ indicate the regimes where the streaks shift past one another trending in opposite directions. In this same flow regime the separation potential between the two larger particle sizes is increasing as the ratio of forces (wall effect to Dean drag) is different between the two sizes.

The dips in separation potential for the passing streaks $(\lambda=0.066$ with both other sizes) shift to lower $\operatorname{Re}_{\mathrm{C}}$ values with the next increase in $\delta$ to 0.0083 . This device is the first device that exhibits excellent separation potential between the $\lambda=0.149$ and $\lambda=0.225$ particles as the $\lambda=0.149$ move into the innermost lateral equilibrium position and the $\lambda=0.225$ do not make that jump until the highest $\operatorname{Re}_{\mathrm{C}}$ values. Finally, at a $\delta$ value of 0.0166 you have high separation potential between all three sizes as the $\lambda=0.149$ particles now start shifting towards the outer half of the channel and the $\lambda=0.225$ particles moving into the inner most lateral equilibrium position. The best separation between all of the particles at the same flow conditions occurs in the regime of the $\delta=0.0166$ device above a $\operatorname{Re}_{\mathrm{C}}$ value of about 200. The other specific separation examples depicted in Figure $3 c$ (i, ii, and iii) are marked on the respective plots.

Case iii in Figure 3 depicts the previously understood fractionation style of Dean flow assisted inertial focusing separation ${ }^{24,27}$. The other two lateral particle arrangements, $i$ and ii, cannot be described by a simple balance of two cross-sectional averaged forces (inertial lift forces and Dean drag).

Three-dimensional behavior and single point focusing. The motivation for this section originates from the lack of a satisfactory answer for the change of equilibrium position with $\delta$ at fixed $\operatorname{Re}_{C}$ and $\lambda$ values. The current understanding of this motion dictates that particles equilibrate to the positions of $z / h= \pm 0.3$ and stay at these height locations due to the stronger shear gradient along the vertical axis compared to across the width. The motion of the streak towards the outer wall is therefore the consequence of the wall interaction lift force of the inner wall which increases faster than the Dean drag force directed in the opposite direction. This makes sense directionally up to a streak position near the centerline but moving past the middle would presumably be stopped by the same effect of the outer wall. Also, if we assume that the wall force is only affected by the $\lambda$ and 
$\operatorname{Re}_{\mathrm{C}}$, the motion of the streak in both directions with increasing $\delta$ (constant $\operatorname{Re}_{\mathrm{C}}$ and $\lambda$ ), first inward towards the center of curvature and then outward at the highest $\delta$ values, should not be possible. One possible explanation would be if the particles were changing position vertically within the channel thereby altering the Dean drag by being exposed to significantly different Dean flow velocities and even different direction. For this reason, a qualitative investigation of the positions of the particle streaks along the height of the channel was performed using high-speed imaging. Particles that are in focus appear as a dark ring around a bright center. A particle further from this focal plane becomes darker in the center the further away it is located until it goes out of focus. Using this concept, the focal plane was set when the top stream of particles came into focus (at the lowest flow rate where focusing was achieved) and images were captured at several different flow conditions for each device. While the analysis is very qualitative it was able to tell the difference between a single streak along the vertical axis versus two distinct streaks.

The dark gray highlighted results described as the transition between the different regimes of focusing in Figures 2 and 3 when $\lambda / \delta^{1 / 2} \sim 2$ turned out to be conditions of note as they are not only the closest approach of the streak to the inside wall of the channel but are indeed conditions at which single z-position (height) focusing is achieved. This type of high quality focusing is important for both high precision positional focusing and due to the correlation between this single point focusing and consistent longitudinal ordering between particles ${ }^{23,36}$. It is also of note that single point particle focusing is possible in simple curved channels not requiring the more complicated structures required for the other known techniques for sheathless alignment of particles ${ }^{26,37,38}$. For the current data set these single point focusing conditions also represent the minimum streak width over the entire parameter space for each particle size $(\lambda=$ 0.066 - Streak Width $\sim 1.36 a, \lambda=0.149$ - Streak Width $\sim 0.91 a, \lambda$ $=0.225-$ Streak Width $\sim 1.01 a$ ). Figure 4 presents exemplar highspeed imaging still frames at different flow conditions verifying the single $\mathrm{z}$-position nature of particle focusing. For $\lambda=0.225$, the transition to single point focusing is most evident in the $\delta=$ 0.0166 device at $\operatorname{Re}_{\mathrm{C}} \approx 150$, for $\lambda=0.149$ in the $\delta=0.0083$ device at $\operatorname{Re}_{\mathrm{C}} \approx 150$, and for $\lambda=0.066$ in the $\delta=0.0008$ device at $\operatorname{Re}_{\mathrm{C}} \approx$ 200. Not all of the smallest particles are forced to a single point in this device, however, the existence of the streak near the inner wall indicates that the equilibrium position is present but perhaps the channel was not long enough for all particles to traverse to this position. It is also possible the curvature ratio is slightly less optimal. Figure $4 \mathrm{~d}$ presents similar high-speed imaging data for the case of $\lambda=0.149$ and $\delta=0.0166$ where the streak of particles instead of continuing to the innermost equilibrium position begins to migrate towards the outer half of the channel never actually changing from two mirrored vertical equilibrium positions. This signifies a complex 3-dimensional motion of the equilibrium position that is dependent on each of the non-dimensional parameters varied in the experiment.

\section{Discussion}

This comprehensive exploration of inertial focusing behavior in curved channels gives insight into the underlying physics. Since the particle distributions can shift in different directions at constant $\operatorname{Re}_{C}$ with increasing curvature, a different mechanism is required to explain the range of equilibrium positions observed. Our hypothesis incorporates a consequence of Dean flow not normally investigated: the addition of curvature to microchannels not only adds Dean drag to the force balance but also changes the shear gradient lift force through the redistribution of the velocity profile ${ }^{39}$. This redistribution caused by Dean flow is significant at the Dean numbers tested in the majority of similar published investigations of separation in Dean flow assisted inertial focusing ( $\mathrm{De}>10$ ). In our hypothesis, we find that a change in vertical position concurrent with the redistribution of the velocity profile with increasing curvature can explain the behaviors presented in this report.

Figure 5 summarizes these effects and the transition between regimes by extrapolating the lateral and vertical equilibrium position results (Figure 1 and Figure 4) to the different curvature ratio devices. In general, the proposed mechanism depends significantly on the previous understanding of inertial lift but improves our understanding of the 3-dimensional variation of the forces in curved channels. As the velocity profile morphs with increasing curvature the shear gradient across the height of the channel on the inner half of the channel drops (left half of surface plots are the inside half of the channel cross section, $\mathrm{x} / w<0.5)$. This drop in shear gradient lift allows particles to have a vertical equilibrium position closer to the centerline at or beyond the zero Dean velocity line at $z / h= \pm 0.25$. By crossing this vertical position the particles experience a switch in the direction of the Dean flow and Dean drag force now directed away from the center of curvature with only the shear gradient lift as a counter balancing force. Considering the decreased shear gradient on the inner half of the channel, compared to the parabolic profile in the $\delta=0$ case, the Dean drag causes the streak to move outwards away from the center of curvature until the shear gradient becomes high enough to counter the Dean drag force. In the other regime where the streaks move towards the center of curvature with increasing $\operatorname{Re}_{C}$, the particles are located in Dean flow directed towards the inner half of the channel. The motion of particles here should scale differently as the countering force is due to the wall effect rather than a nonparabolic velocity profile. The changes in both the lateral and vertical velocity gradients are examined more closely in SI Figures 5 and 6, respectively.

The dependence of this hypothesis on size is also intriguing and is presented graphically in Figure 5b-d. Due to the scaling of the shear gradient lift force with particle size cubed, the smaller particles require a smaller change in the vertical shear gradient in order to move into the oppositely directed Dean flow causing them to move towards the outer half at lower $\delta$ values than the larger particles ${ }^{32}$. The larger particles require a higher $\delta$ value such that the velocity profile is redistributed enough to decrease the vertical shear gradient lift force in such a way to allow for a large enough shift in vertical equilibrium position to have the particles be exposed to the Dean flow directed away from the center of curvature. This will eventually happen for the largest particles as well but did not occur within the current parameter space.

The existence of the flow conditions where single point focusing is achieved are still difficult to explain in these terms without a more in depth understanding of the 3D variation of forces near the inside wall of the channel or without greater $\delta$ resolution. However, it is possible that these special conditions are a small subset of the regime where the shear gradient lift towards the wall is still high enough to force a trajectory of a migrating particle to this innermost equilibrium position. At some point the Dean drag in addition to decreased vertical shear gradient lift force then destabilizes these trajectories and causes the equilibrium position to begin shifting outwards at a different vertical position. It is curious that there seems to be such a large set of operating conditions where this single point focusing behavior is prevalent. Due to the complex positional dependence of inertial lift and Dean drag forces within a single channel cross section ${ }^{18,33,34} \mathrm{a}$ more complete description of the mechanism behind the complex 3 dimensional motion and the transition from inner to outer focusing requires more precise vertical measurement of particle position or full numerical simulation.

With the heightened interest in the application of inertial focusing in curved channels to biomedical applications it is important to elucidate the principles behind the observed behaviors. The two new regimes of focusing discovered through the independent variation of Reynolds number, channel curvature and particle confinement ratio indicate that it is possible with simple curved channels to 

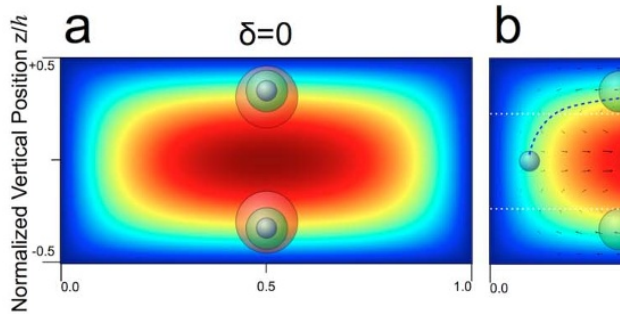

$\delta=0.0008$

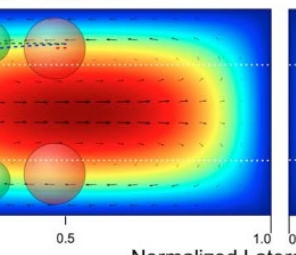

Normalized Lateral Position $\mathrm{x} / \mathrm{w}$

C

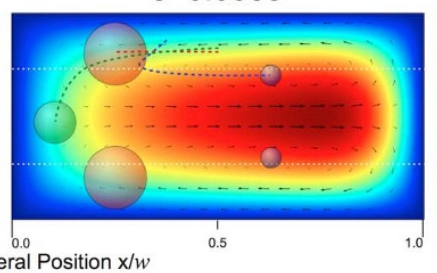

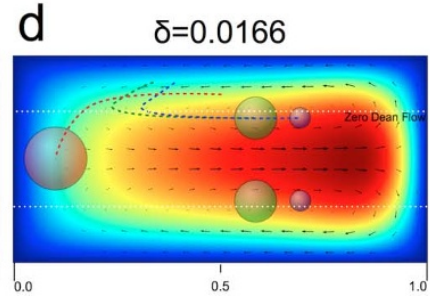

Figure 5 | Graphical representation of the hypothesis for the mechanism of transition between inner and outer focusing in curved microfluidic channels. Simulated velocity profiles with normalized Dean flow vectors are given showing the idealized equilibrium positions (a-d) of the three different sizes of particles $(\lambda=0.066$ blue, $\lambda=0.149$ green, $\lambda=0.225 \mathrm{red})$ in a straight channel $(\delta=0)$ and increasing in curvature left to right. The white dotted lines show the zero Dean flow line where the lateral velocities change direction. The three single point focusing cases and the hypothesized shifting of the equilbrium with increasing $\operatorname{Re}_{\mathrm{C}}$ based upon the data presented in Figure 1 and 4 (dashed lines). The velocity profiles presented are for $\mathrm{Re}_{\mathrm{C}}=419$.

separate particles of an intermediate size (bandpass filtration) and isolate a particle size of choice by varying flow rate in a single device. The transition between these regimes is a size dependent condition at which single point focusing is achieved which other than being the best condition for focusing of a given particle size it will also be a useful case for the study of inter-particle forces in curved inertial focusing flows. In order to explain the complex behaviors witnessed, a balance of forces in 3-dimensions accounting for velocity redistribution due to Dean flow is hypothesized as a function of all of the explored parameters. The guidelines provided herein are a template for future development of the theoretical motion of the equilibrium positions within an arbitrary channel cross-section with secondary flows and their use in future biomedical applications.

\section{Methods}

The designs of the microchannels for independently investigating the effects Reynolds and Dean number on inertial focusing behavior are presented in SI Figure 2. As Dean number is linearly dependent on channel Reynolds number and altering the cross sectional dimensions of a device would also change the particle confinement, the radius of curvature was chosen for adjusting Dean number. Five different channel designs were created over a broad range from the tightest spiral possible to a straight channel. The smallest spiral design was determined by the inlet port size and requiring a minimum distance of a single channel width $(100 \mu \mathrm{m})$ between consecutive lanes of the spiral to ensure bonding. The cross section of each channel is $100 \mu \mathrm{m}$ wide and $50 \mu \mathrm{m}$ high and the length kept constant at $4 \mathrm{~cm}$. The cross sectional dimensions were chosen as a particle of approximate diameter of normal cells (8-15 um in diameter) are all above the stated $a / D_{\mathrm{h}}$ threshold of $0.07^{18}$. These different channels are referred to by their curvature ratio, $\delta$, or the ratio of the hydraulic diameter $\left(D_{h}\right)$ to the average radius of curvature $(r)$, the Reynolds number independent component of the Dean number. The flow rate was varied from $25 \mu \mathrm{L} /$ $\min \left(\mathrm{U}_{\mathrm{Avg}}=0.066 \mathrm{~m} / \mathrm{s}\right)$ to $1200 \mu \mathrm{L} / \mathrm{min}\left(\mathrm{U}_{\mathrm{Avg}}=4 \mathrm{~m} / \mathrm{s}\right)$. Finally, three different particle sizes were tested $(\lambda=0.066, \lambda=0.149$ and $\lambda=0.225)$ in order to investigate the effect of the confinement of particles under the different flow conditions, thereby varying the particle Reynolds number at three different values at an equivalent channel Reynolds number. The complete parameter space $\left(\operatorname{Re}_{\mathrm{C}}, \delta\right.$ and $\left.\lambda\right)$ investigated is therefore summarized in SI Figure 2.

Of note is that the channels used in these studies are fabricated in rigid epoxy with no measurable deformation at the high pressures used during these studies. The importance of the constant cross sectional dimensions along the length of the devices cannot be understated as the deformation of PDMS channels can significantly alter the flow conditions over a large fraction of the channel length ${ }^{40}$. The expansion of the channels alters the balance of Dean drag and inertial lift forces as the larger cross section leads to a higher Dean flow magnitude and the larger cross sectional area decreases the overall velocities and therefore shear gradients in the channel.

PDMS microfluidic channels were fabricated using standard SU-8 photolithography and soft lithography. These channels were then silanized using vapor silanization with tridecafluoro-1,1-2,2-tetrahydrooctyl-trichlorosilane (Gelest) which allows for the molding of PDMS off of the silanized structures. These slianized channels were then used as a template to create a PDMS mold with positive raised features. Molds and silanized channels were separated carefully after 24 hours of curing at $65^{\circ} \mathrm{C}$. Holes were punched into PDMS molds at the inlets and outlets using a $0.75 \mathrm{~mm}$ diameter Harris Uni-Core biopsy punch. Teflon coated wire (0.028 inch diameter, McMaster-Carr) was inserted gently into these holes as to not deform the surface of the PDMS mold. PEEK tubing was roughened near one end using sandpaper and then guided onto teflon coated wire and suspended $\sim 1 \mathrm{~mm}$ from mold surface. Epoxacast 690 (Smooth-On) was mixed and degassed for 30 minutes prior to pouring into the mold. At the same time as molds were filled, slides were coated with epoxy by using a flat PDMS slab and laying 1 inch $\times 3$ inch glass slides onto a drop of epoxy. After $\sim 28$ hours devices were cooled temporarily to $-20^{\circ} \mathrm{C}$ to prevent deformation, the Teflon wire was removed and devices removed from the molds. Then the glass slides were removed from the PDMS slabs and heated to $55^{\circ} \mathrm{C}$ and devices were pressed against slides ensuring bonding.

Water based solutions with $0.1 \%$ Tween $20,0.01 \%$ fluconazole (antifungal) and $0.01 \%$ penicillin-streptomycin (anti-bacterial) were density matched to polystyrene beads using iodixanol (Optiprep, Sigma-Aldrich) to a final fluid density of $1.05 \mathrm{~g} / \mathrm{mL}$ Polystyrene beads (4.4 $\mu \mathrm{m}$ DAPI-fluorescent, Polysciences, $9.9 \mu \mathrm{m}$ FITC-fluorescent, Thermo-Scientific, and $15 \mu \mathrm{m}$, TxRed-fluorescent, Invitrogen) were washed and resuspended in the density-matched solution at a length fraction of $0.1(0.021 \% \mathrm{v} /$ v, $0.103 \% \mathrm{v} / \mathrm{v}$, and $0.230 \% \mathrm{v} / \mathrm{v}$ respectively). Stainless steel syringes (Cole-Parmer) and a high-pressure Harvard Apparatus XPSI syringe pump were used to drive flow through devices (up to $\sim 1000$ psi). A Nikon TiE microscope with a Clara Andor E monochromatic camera at $40 \times$ magnification was used for fluorescence streak imaging. An exposure time of $\sim 1 \mathrm{~s}$ was used and at least 3 images taken and averaged to ensure steady state behavior was captured. High-speed images generated using a Vision Research Phantom v4.2 camera at $4400 \mathrm{fps}$ and $2 \mu$ s exposure. Custom streak analysis software previously described elsewhere was used to obtain streak quality, position and normalized intensity profiles ${ }^{25}$.

Computational modeling efforts were accomplished using COMSOL Multiphysics software using a 2D axisymmetric CFD model with swirl flow to get steady state flow velocities. As the resistance of a curved channel is higher than a straight channel a custom Matlab code was built in order to calculate the correct volume force required to achieve equal average velocities in each of the different curvature cases.

1. Segré, G. \& Silberberg, A. Radial particle displacements in Poiseuille flow of suspensions. Nature 189, 209-210 (1961).

2. Ho, B. \& Leal, L. Inertial migration of rigid spheres in two-dimensional unidirectional flows. J. of Fluid Mech. 65, 365-400 (1974).

3. Segre, G. \& Silberberg, A. Behavior of macroscopic rigid spheres in Poiseuille flow. J. Fluid Mech. 14, 115-135 (1961).

4. Amini, H. et al. Engineering fluid flow using sequenced microstructures. Nature Communications 4, 1-8 (2013).

5. Matas, J.-P., Morris, J. F. \& Guazzelli, É. Inertial migration of rigid spherical particles in Poiseuille flow. J. Fluid Mech. 515, 171-195 (2004).

6. Di Carlo, D., Irimia, D., Tompkins, R. \& Toner, M. Continuous inertial focusing, ordering, and separation of particles in microchannels. Proceedings of the National Academy of Sciences 104, 18892-18897 (2007).

7. Bhagat, A., Kuntaegowdanahalli, S. \& Papautsky, I. Enhanced particle filtration in straight microchannels using shear-modulated inertial migration. Physics of Fluids 20, 1-4 (2008).

8. Zhou, J., Giridhar, P. V., Kasper, S. \& Papautsky, I. Modulation of aspect ratio for complete separation in an inertial microfluidic channel. Lab on a Chip 13, 1919-1929 (2013).

9. Xiang, N. et al. Quantitative characterization of the focusing process and dynamic behavior of differently sized microparticles in a spiral microchannel. Microfluidics and Nanofluidics 14, 89-99 (2012).

10. Wu, L., Guan, G., Hou, H., Bhagat, A. \& Han, J. Separation of Leukocytes From Blood Using Spiral Channel With Trapezoid Cross-section. Analytical Chemistry 84, 9324-9331 (2012).

11. Hur, S. C., Choi, S. E., Kwon, S. \& Di Carlo, D. Inertial focusing of non-spherical microparticles. Applied Physics Letters 99, 044101 (2011).

12. Masaeli, M. et al. Continuous Inertial Focusing and Separation of Particles by Shape. Physical Review X 2, 031017 (2012).

13. Gossett, D. R. et al. Inertial Manipulation and Transfer of Microparticles Across Laminar Fluid Streams. Small 8, 2757-2764 (2012).

14. Mach, A. J., Kim, J. H., Arshi, A., Hur, S. C. \& Di Carlo, D. Automated cellular sample preparation using a Centrifuge-on-a-Chip. Lab on a Chip 11, 2827-2834 (2011).

15. Gossett, D. R. et al. Hydrodynamic stretching of single cells for large population mechanical phenotyping. Proceedings of the National Academy of Sciences 109, 7630-7635 (2012). 
16. Hou, H. W. et al. Isolation and retrieval of circulating tumor cells using centrifugal forces. Scientific Reports 3, 1259 (2013).

17. Ozkumur, E. et al. Inertial Focusing for Tumor Antigen-Dependent and Independent Sorting of Rare Circulating Tumor Cells. Science Translational Medicine 5, 179ra47 (2013).

18. Di Carlo, D. Inertial microfluidics. Lab on a Chip 9, 3038-3046 (2009).

19. Bhagat, A., Kuntaegowdanahalli, S. \& Papautsky, I. Continuous particle separation in spiral microchannels using dean flows and differential migration. Lab on a Chip 8, 1906-1914 (2008).

20. Di Carlo, D., Edd, J., Irimia, D., Tompkins, R. \& Toner, M. Equilibrium separation and filtration of particles using differential inertial focusing. Analytical Chemistry 80, 2204-2211 (2008).

21. Gossett, D. R. \& Di Carlo, D. Particle Focusing Mechanisms in Curving Confined Flows. Analytical Chemistry 81, 8459-8465 (2009).

22. Guan, G. et al. Spiral microchannel with rectangular and trapezoidal crosssections for size based particle separation. Scientific Reports 3, 1475 (2013)

23. Kemna, E. et al. High-yield cell ordering and deterministic cell-in-droplet encapsulation using Dean flow in a curved microchannel. Lab on a Chip 12, 2881-2887 (2012)

24. Kuntaegowdanahalli, S. S., Bhagat, A. A. S., Kumar, G. \& Papautsky, I. Inertial microfluidics for continuous particle separation in spiral microchannels. Lab on a Chip 9, 2973-2980 (2009).

25. Martel, J. \& Toner, M. Inertial focusing dynamics in spiral microchannels. Physics of Fluids 24, 032001 (2012)

26. Oakey, J., Applegate, R. Jr., Arellano, E. \& Di Carlo, D. Particle Focusing in Staged Inertial Microfluidic Devices for Flow Cytometry. Anal. Chem. 82, 3862-3867 (2010)

27. Russom, A. et al. Differential inertial focusing of particles in curved low-aspectratio microchannels. New Journal of Physics 11, 075025 (2009).

28. Seo, J., Lean, M. \& Kole, A. Membrane-free microfiltration by asymmetric inertial migration. Applied Physics Letters 91, 033901 (2007).

29. Seo, K. W., Choi, Y. S. \& Lee, S. J. Dean-coupled inertial migration and transient focusing of particles in a curved microscale pipe flow. Experiments in Fluids 53, 1867-1877 (2012)

30. Hasni, A. et al. Focusing and Sorting of Particles in Spiral Microfluidic Channels. Procedia Engineering 25, 1197-1200 (2011).

31. Xiang, N. et al. High-throughput inertial particle focusing in a curved microchannel: Insight into the flow-rate mechanism and process model. Biomicrofluidics 7, 044116 (2013).

32. Di Carlo, D., Edd, J., Humphry, K., Stone, H. \& Toner, M. Particle Segregation and Dynamics in Confined Flows. Physical Review Letters 102, 094503 (2009).

33. Zhou, J. \& Papautsky, I. Fundamentals of inertial focusing in microchannels. Lab on a Chip 13, 1121-1130 (2013).
34. Asmolov, E. The inertial lift on a spherical particle in a plane Poiseuille flow at large channel Reynolds number. J. of Fluid Mech. 381, 63-87 (1999).

35. Ciftlik, A., Ettori, M. \& Gijs, M. High throughput-per-footprint intertial focusing Small 9, 2764-2771 (2013).

36. Lee, W., Amini, H., Stone, H. A. \& Di Carlo, D. Dynamic self-assembly and control of microfluidic particle crystals. Proceedings of the National Academy of Sciences 107, 22413-22418 (2010).

37. Chung, A. et al. Microstructure-induced helical vortices allow single-stream and long-term inertial focusing. Lab on a Chip 13, 2942-2949 (2013).

38. Chung, A., Gossett, D. R. \& Di Carlo, D. Three Dimensional, Sheathless, and High-Throughput Microparticle Inertial Focusing Through Geometry- Induced Secondary Flows. Small 9, 685-690 (2012).

39. Vriend, H. J. D. Velocity redistribution in curved rectangular channels. Journal of Fluid Mechanics 107, 423-439 (1981).

40. Gervais, T., El-Ali, J., Günther, A. \& Jensen, K. Flow-induced deformation of shallow microfluidic channels. Lab on a Chip 6, 500-507 (2006).

\section{Acknowledgments}

Joseph M. Martel is a National Science Foundation Graduate Research Fellow. This work was supported in part by the National Institute of Biomedical Imaging and Bioengineering (BioMEMS Resource Center, P41 EB002503). Thanks to Ian Wong, Dino Di Carlo and Jon Edd for helpful discussion, Eugene Lim, Salil Desai and Bashar Hamza for epoxy device fabrication development.

\section{Author contributions}

J.M.M. designed and ran experiments, prepared figures and wrote main text. M.T. assisted with figure design and both authors reviewed manuscript.

\section{Additional information}

Supplementary information accompanies this paper at http://www.nature.com/ scientificreports

Competing financial interests: The authors declare no competing financial interests. How to cite this article: Martel, J.M. \& Toner, M. Particle Focusing in Curved Microfluidic Channels. Sci. Rep. 3, 3340; DOI:10.1038/srep03340 (2013).

This work is licensed under a Creative Commons Attribution-

NonCommercial-NoDerivs 3.0 Unported license. To view a copy of this license, visit http://creativecommons.org/licenses/by-nc-nd/3.0 